\title{
LOW-MASS DIFFRACTION DISSOCIATION AT THE LHC. ROLE OF THE BACKGROUND
}

\author{
L.L. JENKOVSZKY, ${ }^{1}$ O.E. KUPRASH, ${ }^{2}$ V.K. MAGAS ${ }^{3}$ \\ ${ }^{\mathbf{1}}$ Bogolyubov Institute for Theoretical Physics, Nat. Acad. of Sci. of Ukraine \\ (14b, Metrolohichna Str., Kyiv 03680, Ukraine) \\ ${ }^{2}$ Taras Shevchenko National University of Kyiv, Nuclear Physics Department \\ (6, Academician Glushkov Ave., Kyiv 03022, Ukraine) \\ ${ }^{3}$ Departament d'Estructura i Constituents de la Matéria \\ (Universitat de Barcelona, Diagonal 647, Barcelona 08028, Spain)
}

\begin{abstract}
A dual model with a nonlinear proton Regge trajectory in the missing mass $\left(M_{X}^{2}\right)$ channel is constructed. A background based on a direct-channel exotic trajectory, developed and applied earlier for the inclusive electron-proton cross section description in the nucleon resonance region, is used. The parameters of the model are determined from the extrapolations to earlier experiments. Predictions for the low-mass $\left(2<M_{X}^{2}<8 \mathrm{GeV}^{2}\right)$ diffraction dissociation cross sections at the LHC energies are given.
\end{abstract}

\section{Introduction}

Experimentally, the diffraction dissociation in protonproton scattering was intensively studied in the 1970s at the Fermilab and the CERN ISR [1-3]. In particular, the double differential cross section $\frac{d \sigma}{d t d M_{X}^{2}}$ was measured in the region $0.024<-t<0.234(\mathrm{GeV} / \mathrm{c})^{2}, \quad 0<M^{2}<$ $0.12 s$, and $(105<s<752) \mathrm{GeV}^{2}$, see [3], and a single peak in $M_{X}^{2}$ was identified.

The low-mass single diffraction dissociation (SDD) of protons $p p \rightarrow p X$, as well as their double diffraction dissociation (DDD), are among the priorities at the LHC. For the CMS Collaboration, the SDD mass coverage is presently limited to some $10 \mathrm{GeV}$. With the Zero Degree Calorimeter (ZDS), this could be reduced to smaller masses, in case the SDD system produces very forward neutrals, i.e. like a $N^{*}$ decaying into a fast leading neutron. Together with the T2 detectors of TOTEM, SDD masses down to $4 \mathrm{GeV}$ could be covered. This is not the case until the TOTEM trigger (data acquisition) system is combined together with the CMS ones. This is not likely before the year 2012 shut down. In principle, ATLAS can do a similar improvement, since the LHC lay-out at the distance of our proposed Forward Shower Counters' (FSC) locations is similar. ALICE and LHCb have different beam arrangements, but their acceptances for central diffraction (double pomeron exchange) were also investigated (see, e.g., [4])

While high-mass diffraction dissociation receives much attention, mainly due to its relatively easy theoretical treatment within the triple Reggeon formalism [5$8]$ and the successful reproduction of the data $[5,10]$, this is not the case for low masses, which are beyond the range of perturbative quantum chromodynamics (QCD). The forthcoming measurements at the LHC urge a relevant theoretical understanding and treatment of lowmass DD, which essentially has both spectroscopic and dynamic aspects. The low-mass $M_{X}$ spectrum is rich of nucleon resonances. Their discrimination is a difficult experimental task, and theoretical predictions of the appearance of the resonances depending on $s, t$, and $M$ are also very difficult since, as mentioned, perturbative QCD or the asymptotic Regge pole formula is of no use here. In this paper, as well as in the recent work [9], we try to partially fill this gap, attacking the problem by means of the dual-Regge approach to the inelastic form factor (production amplitude). We concentrate on the single diffraction dissociation; a generalization to DDD is straightforward.

Diffraction, elastic and inelastic, in the LHC energy range is dominated by a single Pomeron exchange in the $t$ channel (see, e.g., $[11,12]$ ), enabling the use of 

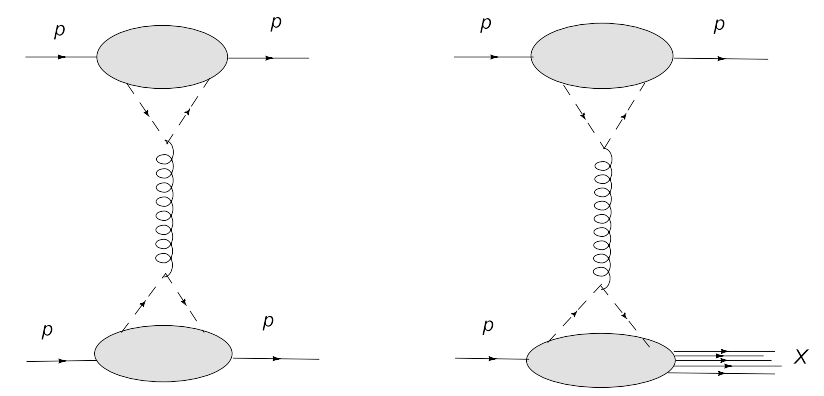

Fig. 1. Figure caption: Elastic scattering (left panel) and diffraction dissociation (right panel) in a model with a pomeron exchange coupled to the proton by quarks

Regge factorization, Fig. 1. Accordingly, the knowledge of two vertices and the Regge propagator is essential for the construction of the scattering amplitude. Relying on the known properties of the elastic proton-Pomeronproton vertex and by adopting a simple supercritical Pomeron pole exchange (propagator) in the $t$ channel, we concentrate on the construction of a proper inelastic proton-Pomeron- $M_{X}$ vertex, the central object of our study. The solution of this problem became possible, to a large extent, due to the similarity between the inelastic $\gamma^{*} p \rightarrow M_{x}$ and Pomeron + proton $\rightarrow M_{x}$ vertices. We will extensively use the earlier results on the $\gamma^{*} p \rightarrow M_{x}$ transition, successfully applied to the JLab data $[14,15]$ in constructing the lower, Pomeron + proton $\rightarrow M_{x}$ vertices of Fig. 1, right panel. In doing so, we draw a parallel between the virtual photon and the Pomeron. Apart from their opposite $C$ parities, they are very similar. However, one should take into account changes in the kinematics, namely the photon virtuality (e.g., at the JLab) $Q^{2}=-q^{2}$ of Fig. 2 becomes the squared momentum transfer $-t$ of Fig. 1 , and the energy variable, $s$, has to replaced by the missing mass, $M_{X}^{2}$ :

$W_{2}\left(q^{2}, s\right)_{\gamma^{*} p \rightarrow N_{i}^{*}, \Delta}($ at JLab $) \Rightarrow$

$\Rightarrow W_{2}\left(t, M^{2}\right)_{P p \rightarrow N^{*}}($ at the LHC $)$.

The unknown inelastic form factor of the type shown in Fig. 2 is related, by the optical theorem, to the imaginary part of the forward $\gamma^{*}(P)-p$ scattering amplitude. Following $[14,15,17]$, we use a dual amplitude for this reaction, in its low-energy (here $M_{X}^{2}$ ) resonance region, dominated by the contribution of relevant direct-channel trajectories. The correct choice of these trajectories is a crucial point in our approach. In the case of $\gamma^{*} p$ scattering (e.g., JLab) these were the $N^{*}$ and $\Delta$ trajectories, see $[14,15,17]$. Here, instead, by quantum numbers, the

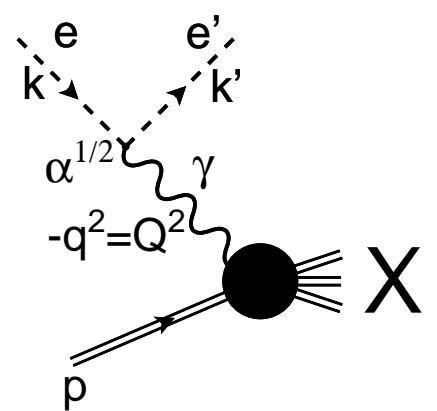

Fig. 2. Virtual photon + proton $\rightarrow M_{X}$ transition

relevant direct channel trajectory is that of the proton, to be parametrized in Sec. 5 .

In principle, one could proceed by counting the resonances one-by-one. However, apart from the technical complexity of counting the single resonances, there is also a conceptual advantage in the Regge-dual approach: Regge trajectories and, more generally, dual models comprise the dynamics in a complete and continuous way, thus opening the way to study and relate different reactions in any kinematic region. Examples are the finitemass sum rules, contained in the present formalism automatically. One more important point: the advantage of using the dual-Regge model with a non-linear Regge trajectory presented in this paper over a one-to-one account for the particular resonances is that it automatically takes care of the relative weight of each resonance and extrapolates to higher masses with a limited number of resonances on any trajectory.

\section{Elastic Scattering}

The $p p$ scattering amplitude corresponding to Fig. 1 (left) is [7]

$A(s, t)_{P}=$

$-\beta^{2}\left[f^{u}(t)+f^{d}(t)\right]^{2}\left(\frac{s}{s_{0}}\right)^{\alpha_{P}(t)-1} \frac{1+e^{-i \pi \alpha_{P}(t)}}{\sin \pi \alpha_{P}(t)}$,

where $f^{u}(t)$ and $f^{d}(t)$ are the amplitudes for the emission of $u$ and $d$ valence quarks by the nucleon, $\beta$ is the quark-Pomeron coupling, to be determined below; $\alpha_{P}(t)$ is a vacuum Regge trajectory. It is assumed [7] that the Pomeron couples to the proton via quarks like a scalar photon.

A single-Pomeron exchange of Eq. (15) is valid at the LHC energies; however, at lower energies (e.g., those of the ISR or the SPS), the contribution of non-leading 
Regge exchanges should be accounted for as well. In other words, instead of Eq. (15), one should use the sum

$A(s, t)_{p p}^{\bar{p} p}=P+f \pm(O+\omega)$,

where $P$ stands for the Pomeron contribution, Eq. (15), $O$ is that of the odderon (Pomeron's odd- $C$ counterpart), and $f, \omega$ symbolize the contribution from relevant secondary reggeons. Note that the even- $C$ contributions enter with equal signs in $\bar{p} p$ scattering and with alternating signs in $p p$ (for more details, see [12] and earlier references therein).

Below, following [13], we include only the leading Pomeron contribution $P$ (see Eq. 15).

Thus, the unpolarized elastic $p p$ differential cross section is

$$
\frac{d \sigma}{d t}=\frac{\left[3 \beta F^{p}(t)\right]^{4}}{4 \pi \sin ^{2}\left[\pi \alpha_{P}(t) / 2\right]}\left(s / s_{0}\right)^{2 \alpha_{P}(t)-2} .
$$

The norm $\beta$ appearing in Eq. (15) was found in [7] from the forward elastic scattering, $d \sigma / d t \approx 80 \mathrm{mb} / \mathrm{GeV}^{2}$ at $\sqrt{s}=23.6$ and $30.8 \mathrm{GeV}$, resulting, at the unit Pomeron intercept, $\alpha_{P}(0)=1$, in $\beta^{4} /(4 \pi) \approx 1 \mathrm{mb} / \mathrm{GeV}^{2}$ [7].

To account for the rise of the cross sections, following the model and fits of Donnachie and Landshoff, see [11] and earlier references therein, we use a Pomeron trajectory whose intercept is slightly beyond one, namely, $\alpha_{P}(0)=1.08$ providing for excellent fits to the total cross sections [11]. However, the extrapolation with such an intercept and the input value of $\beta$ strongly overshoots the elastic forward cross section measured at higher energies, e.g. $\sqrt{s}=1800 \mathrm{GeV}$ [18]. There are several reasons for this inconsistency. One is that, at the normalization point, 23.6 or $30.6 \mathrm{GeV}$, the contribution from secondary Reggeons and/or a constant background should be included. In what follows, we use the Pomeron trajectory of the form (see [11]) $\alpha_{P}(t)=1.08+0.25 t$ and consequently relax the above norm of $\beta$. Instead, it will be included in the overall normalization factor of the amplitude/cross section $A_{0}$ that absorbs also the parameter $a$ of Eq. (16) from Section 6.

Another important issue is the neglect of absorption (unitary) corrections. As an efficient way to avoid any conflict with unitarity, a dipole Pomeron pole can be used instead of (15) (see [12]). We intend to come back in a forthcoming investigation to the study of the role of the subleading reggeons and of the absorption corrections.

A dipole form can be used for the form factor,

$$
F^{p}(t)=\frac{4 m^{2}-2.9 t}{4 m^{2}-t} \frac{1}{(1-t / 0.71)^{2}},
$$

where $m$ is the proton mass.

\section{Single Diffraction Dissociation (SDD)}

In the single diffraction dissociation, a system $X$ with missing mass $M_{X}$ is produced at small $|t|$. At sufficiently large $s / M_{X}^{2}$, which is the case at the LHC, the process is dominated by a Pomeron exchange. This case was treated in [7] for missing masses beyond the resonance region and in [23] in the resonance region. For large missing masses, the triple Regge limit applies $[6,10,16$, 24]. Although the large- $M_{X}$ diffraction dissociation is outside the scope of the present paper, we mention it below, in particular in connection with duality relations called the finite-mass sum rule that relate low- and highmissing-mass dynamics.

Similar to the case of elastic scattering (Sec. 2), the double differential cross section for the SDD reaction can be written with the Regge factorization as [13]

$$
\frac{d^{2} \sigma}{d t d M_{X}^{2}}=\frac{9 \beta^{4}\left[F^{p}(t)\right]^{2}}{4 \pi \sin ^{2}\left[\pi \alpha_{P}(t) / 2\right]}\left(s / M_{X}^{2}\right)^{2 \alpha_{P}(t)-2} \times
$$

$$
\times\left[\frac{W_{2}}{2 m}\left(1-M_{X}^{2} / s\right)-m W_{1}\left(t+2 m^{2}\right) / s^{2}\right],
$$

where $W_{i}, \quad i=1,2$ are related to the structure functions of the nucleon, and $W_{2} \gg W_{1}$. For high $M_{X}^{2}$, the $W_{1,2}$ are Regge-behaved, while their behavior for small $M_{X}^{2}$ is dominated by nucleon resonances. Thus, the behavior of (5) in the low-missing-mass region depends to a large extent on the transition form factors or resonance structure functions. The knowledge of the inelastic form factors (or transition amplitudes) is crucial for the calculation of low-mass diffraction dissociation from Eq. (5). We introduce these transition amplitudes in the next section.

At large $s$ (the LHC energies), one can safely neglect terms $M_{X}^{2} / s$ and $\left(t+2 m^{2}\right) / s$ in Eq. (5). Furthermore, we have replaced the familiar form of the signature factor in the amplitude, $\frac{1+e^{-i \pi \alpha_{P}(t)}}{\sin \pi \alpha_{P}(t)}$ used in [7], by a simple exponential one $e^{-i \pi \alpha_{P}(t) / 2}$. For the proton elastic form factor $F^{p}(t)$, Eq. (4), we use a dipole form

$F^{p}(t)=(1-t / 0.71)^{-2}$

(note that we neglect the first factor of Eq. (4) producing a break in the small $|t|$ behavior of the elastic differential cross section).

Hence, Eq. (5) simplifies in the LHC energy region to

$$
\frac{d^{2} \sigma}{d t d M_{X}^{2}} \approx \frac{9 \beta^{4}\left[F^{p}(t)\right]^{2}}{4 \pi}\left(s / M_{X}^{2}\right)^{2 \alpha_{P}(t)-2} \frac{W_{2}}{2 m} .
$$


Equations (5) and (7) do not contain the elastic scattering limit because the inelastic form factor $W_{2}\left(M_{X}, t\right)$ has no elastic form factor limit $F(t)$ as $M_{X} \rightarrow m$. This problem is similar to the $x \rightarrow 1$ limit of the deep inelastic structure function $F_{2}\left(x, Q^{2}\right)$. The elastic contribution to SDD should be added separately, as discussed below in Sec. 6. To be sure, we eliminate this region in the present work by imposing $M_{X}^{2}>2 \mathrm{GeV}^{2}$.

\section{Dual-Regge Model of the Inelastic Form Factors (Transition Amplitudes)}

The main idea behind the present work is the Regge-dual connection between the inelastic form factor (see Fig. 2) appearing in the lower vertex of Fig. 1 and the directchannel low-energy (here, $M_{X}^{2}$ ) dual amplitude [9], as illustrated in Fig. 3.

Figure 3 shows the connection between the inelastic form factor (structure function) appearing in the lower vertex of the left panel of Fig. 1, via duality, unitarity (generalized optical theorem), and Veneziano-duality, and its direct channel, resonance decomposition (rightmost term of Fig. 3).

The invariant dual on-shell scattering amplitude with Mandelstam analyticity (DAMA), applicable both to the diffractive and non-diffractive components, reads $[14,15$, $17,19]$

$D(s, t)=\int_{0}^{1} d z\left(\frac{z}{g}\right)^{-\alpha\left(s^{\prime}\right)-1}\left(\frac{1-z}{g}\right)^{-\alpha\left(t^{\prime}\right)-1}$,

where $s^{\prime}=s(1-z), t^{\prime}=t z(s, t$ are the Mandelstam variables); and $g$ is a model parameter, $g>1$.

For $s \rightarrow \infty$ and fixed $t$, it has the Regge asymptotic behavior

$D(s, t) \approx \sqrt{\frac{2 \pi}{\alpha_{t}(0)}} g^{1+a+i b}\left(\frac{s \alpha^{\prime}(0) g \ln g}{\alpha_{t}(0)}\right)^{\alpha_{t}(0)-1}$

where $a=\operatorname{Re} \alpha\left(\frac{\alpha_{t}(0)}{\alpha^{\prime}(0) \ln g}\right)$ and $b=\operatorname{Im} \alpha\left(\frac{\alpha_{t}(0)}{\alpha^{\prime}(0) \ln g}\right)$.

Contrary to the Veneziano model, DAMA [19] not only allows for, but rather requires the use of nonlinear complex trajectories providing the resonance widths via the imaginary part of the trajectory and, in a special case of the restricted real part of the trajectory, resulting in a finite number of resonances. More specifically, the asymptotic rise of the trajectories in DAMA is limited by the important upper bound

$\left|\frac{\alpha(s)}{\sqrt{s} \ln s}\right| \leq$ const, $\quad s \rightarrow \infty$.

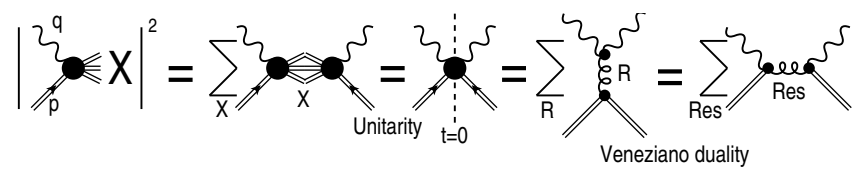

Fig. 3. Connection, through unitarity (generalized optical theorem) and Veneziano-duality, between the inelastic form factor and the sum of direct-channel resonances

The pole structure of DAMA is similar to that of the Veneziano model, except that multiple poles appear on daughter levels $[14,15,17,19]$,

$D(s, t)=\sum_{n=0}^{\infty} g^{n+1} \sum_{l=0}^{n} \frac{\left[-s \alpha^{\prime}(s)\right]^{l} C_{n-l}(t)}{[n-\alpha(s)]^{l+1}}$,

where $C_{n}(t)$ is the residue, whose form is fixed by the $t$-channel Regge trajectory (see [19])

$C_{l}(t)=\frac{1}{l !} \frac{d^{l}}{d z^{l}}\left[\left(\frac{1-z}{g}\right)^{-\alpha_{t}(t z)}\right]_{z=0}$

The presence of the multipoles, Eq. (10), does not contradict the theoretical postulates. On the other hand, they can be removed without any harm to the dual model by means of the so-called Van der Corput neutralizer [19], resulting in a "Veneziano-like" pole structure:

$D(s, t)=\sum_{n=0}^{\infty} \frac{C_{n}(t)}{n-\alpha_{s}(s)}$.

We disregard the symmetry (spin and isospin) properties of the problem, concentrating on its dynamics.

\section{1. $Q^{2}$-dependence $\Rightarrow t$-dependence of the Dual Amplitude}

We recall that $Q^{2}$ (photon virtuality in electroproduction) will be replaced below by $-t$ (Pomeron "virtuality"). The main problem is how to introduce the $Q^{2}$-dependence in the dual model, matching its Regge asymptotic behavior and pole structure to standard forms known from the literature. (This is the famous problem of the off-mass-shall continuation of the $S$ matrix.) Note that any correct identification of this $Q^{2}$ dependence in a single asymptotic limit of the dual amplitude, by duality, will extend it to other kinematic regions. In $[14,15,17]$, a solution combining the Regge behavior and Bjorken scaling limits of the structure functions (or $Q^{2}$-dependent $\gamma^{*} p$ cross sections) was suggested (for an alternative solution see [20]).

For our purposes, i.e. for low-mass SDD, the directchannel pole decomposition of the dual amplitude (12) is 
relevant. Anticipating its application in SDD, we write it as [9]

$$
A\left(M_{X}^{2}, t\right)=a \sum_{n=0,1, \ldots} \frac{f(t)^{2(n+1)}}{2 n+0.5-\alpha\left(M_{X}^{2}\right)},
$$

where $\alpha\left(M_{X}^{2}\right)$ is a non-linear Regge trajectory in the Pomeron-proton system, $t$ is the squared transfer momentum in the $P p \rightarrow P p$ reaction, and $a$ is the normalization factor, which will be absorbed together with $\beta$ in the overall normalization coefficient $A_{0}$ to be fitted to the data, see Sec. 6 . We recall once again that $M_{X}^{2}$ replaces $s$ here (the direct, $P p$ channel "energy").

The form factor $f(t)$ appearing in the $P p \rightarrow P p$ system should not be confused with $F^{p}(t)$ in the $p p P$ vertex. It is fixed by the dual model $[14,15,17,20]$, in particular by the compatibility of its Regge asymptotics with Bjorken scaling [14, 15, 17] and reads

$f(t)=\left(1-t / t_{0}\right)^{-2}$,

where $t_{0}$ is a parameter to be fitted to the data, for example, by comparing the height of the resonance peaks for different $t$. However, since, for the moment, we have no data on differential SDD cross section, we set $t_{0}=$ $0.71 \mathrm{GeV}^{2}$ for simplicity, as in the proton elastic elastic form factor, Eq. (4) [9].

Note that this form factor in Eq. (13) enters with a power $2(n+1)$ strongly damping the contributions of higher spin resonances ${ }^{1}$.

The inelastic form factor in the diffraction dissociation is similar to that in $\gamma^{*} p$, treated in [15] up to the replacement of the photon by a Pomeron, whose parity is different from that of the photon. As a consequence, we have a single direct-channel resonance trajectory, that of the proton, plus the exotic, nonresonance trajectory providing the background dual to the Pomeron exchange in the cross channel. The proton trajectory was studied in details in [21] and will be introduced in the next section.

Then we proceed:

$$
\begin{aligned}
& \nu W_{2}\left(M_{X}^{2}, t\right)=F_{2}(x, t)= \\
& =\frac{4(-t)(1-x)^{2}}{\alpha\left(M_{x}^{2}-m^{2}\right)\left(1+4 m^{2} x^{2} /(-t)\right)^{3 / 2}} \operatorname{Im} A\left(M_{X}^{2}, t\right),
\end{aligned}
$$

\footnotetext{
1 In an alternative approach in [20], the form factors enter with the same power for all the resonances on a given trajectory. The advantage of the models with increasing powers of the form factors is that the poorly known high spin resonances are strongly suppressed and thus do not affect the final results.
}

where $\alpha$ is a fine structure constant, $\nu$ is defined via $2 m \nu=M_{x}^{2}-m^{2}-t$, and $x=\frac{-t}{2 m \nu}$ is the Bjorken variable.

The imaginary part of the transition amplitude reads

$\operatorname{Im} A\left(M_{X}^{2}, t\right)=$

$=a \sum_{n=0,1, \ldots} \frac{[f(t)]^{2(n+1)} \operatorname{Im} \alpha\left(M_{x}^{2}\right)}{\left(2 n+0.5-\operatorname{Re} \alpha\left(M_{X}^{2}\right)\right)^{2}+\left(\operatorname{Im} \alpha\left(M_{X}^{2}\right)\right)^{2}}$.

Next we insert the proton trajectory $\alpha\left(M_{X}^{2}\right)$ into Eq. (16), and subsequently into Eq. (7). The explicit expression for the proton trajectory and the values of parameters are presented in the next section. For more details, see also [21].

\section{The Proton Trajectory in the $M_{X}^{2}$-channel}

The Pomeron-proton channel, $P p \rightarrow M_{X}^{2}$ (see the lower part of Fig. 1, right panel), couples to the proton trajectory, with the $I\left(J^{P}\right)$ resonances: $1 / 2\left(5 / 2^{+}\right), F_{15}, m=$ $1680 \mathrm{MeV}, \Gamma=130 \mathrm{MeV} ; 1 / 2\left(9 / 2^{+}\right), H_{19}, m=2200$ $\mathrm{MeV}, \Gamma=400 \mathrm{MeV}$; and $1 / 2\left(13 / 2^{+}\right), K_{1,13}, m=2700$ $\mathrm{MeV}, \Gamma=350 \mathrm{MeV}$. The status of the first two is firmly established [22], while the third one, $N^{*}(2700)$, is less certain, with its width varying between $350 \pm 50$ and $900 \pm 150 \mathrm{MeV}[22]$. Still, with the stable proton included, we have a fairly rich trajectory, $\alpha\left(M^{2}\right)$, whose real part is shown in Fig. 4.

Despite the seemingly linear form of the trajectory, it is not that: the trajectory must contain an imaginary part corresponding to the finite widths of the resonances on it. The non-trivial problem of combining the nearly linear real function with its imaginary part was solved in [21] by means of dispersion relations.

We use the explicit form of the trajectory derived in [21], ensuring the correct behavior of both its real and imaginary parts. The imaginary part of the trajectory can be written as

$\operatorname{Im} \alpha(s)=s^{\delta} \sum_{n} c_{n}\left(\frac{s-s_{n}}{s}\right)^{\lambda_{n}} \theta\left(s-s_{n}\right)$,

where $\lambda_{n}=\operatorname{Re} \alpha\left(s_{n}\right)$. Equation (17) has the correct threshold behavior, while the analyticity requires that $\delta<1$. The boundedness of $\alpha(s)$ for $s \rightarrow \infty$ follows from the condition that the amplitude, in the Regge form, should have no essential singularity at infinity in the cut plane. 


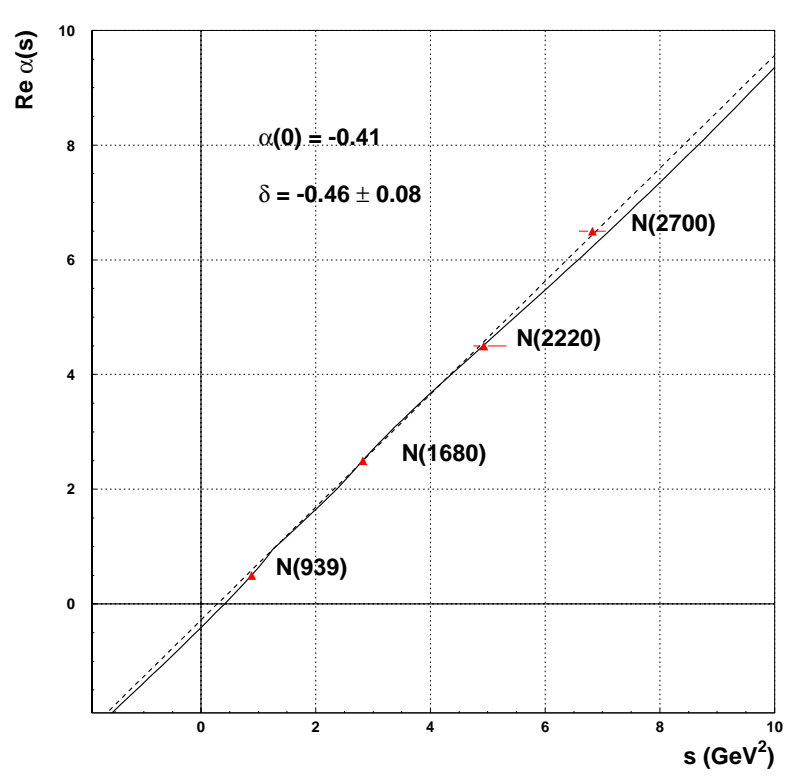

Fig. 4. The real part of the proton Regge trajectory. The dashed line corresponds to the result of a linear fit, the solid line is the fit from [21]

The real part of the proton trajectory is given by

$\operatorname{Re} \alpha(s)=\alpha(0)+\frac{s}{\pi} \sum_{n} c_{n} \mathcal{A}_{n}(s)$,

where

$$
\begin{aligned}
& \mathcal{A}_{n}(s)=\frac{\Gamma(1-\delta) \Gamma\left(\lambda_{n}+1\right)}{\Gamma\left(\lambda_{n}-\delta+2\right) s_{n}^{1-\delta}} \times \\
& \times{ }_{2} F_{1}\left(1,1-\delta ; \lambda_{n}-\delta+2 ; \frac{s}{s_{n}}\right) \theta\left(s_{n}-s\right)+ \\
& +\left\{\pi s^{\delta-1}\left(\frac{s-s_{n}}{s}\right)^{\lambda_{n}} \cot [\pi(1-\delta)]-\right. \\
& \left.-\frac{\Gamma(-\delta) \Gamma\left(\lambda_{n}+1\right) s_{n}^{\delta}}{s \Gamma\left(\lambda_{n}-\delta+1\right)} F_{1}\left(\delta-\lambda_{n}, 1 ; \delta+1 ; \frac{s_{n}}{s}\right)\right\} \times
\end{aligned}
$$$$
\times \theta\left(s-s_{n}\right) .
$$

As was already mentioned, the proton trajectory, also called $\mathrm{N}^{+}$trajectory [21], contains the baryons $\mathrm{N}(939)$ $\frac{1}{2}^{+}, \mathrm{N}(1680) \frac{5}{2}^{+}, \mathrm{N}(2220) \frac{9}{2}^{+}$, and $\mathrm{N}(2700) \frac{13}{2}^{+}[22]$. In the fit, the input data are the masses and widths of

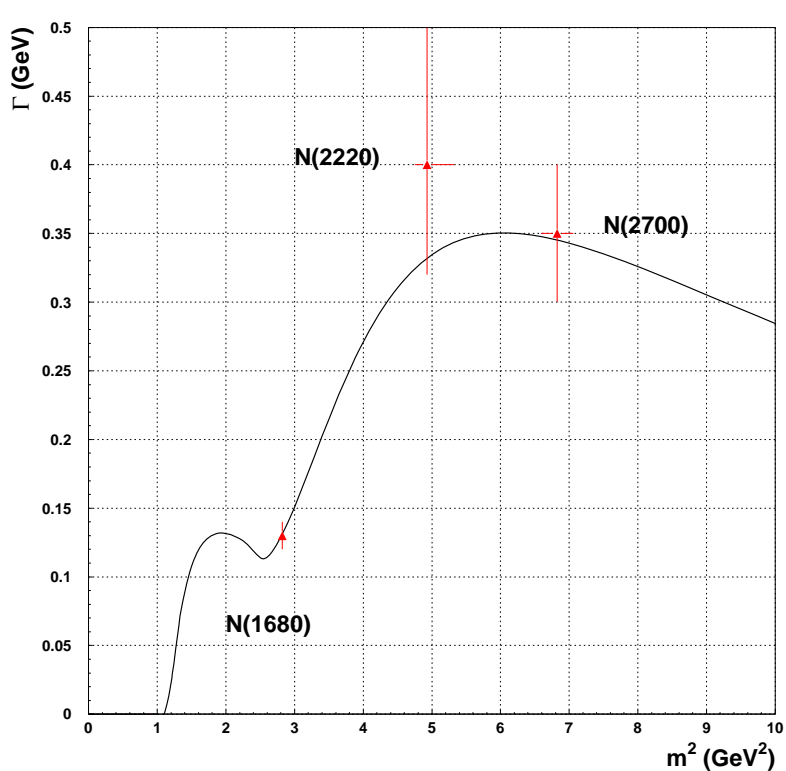

Fig. 5. Widths of the resonances, $\Gamma=\frac{\operatorname{Im} \alpha\left(M^{2}\right)}{M \operatorname{Re} \alpha^{\prime}\left(M^{2}\right)}$, appearing on the proton trajectory, calculated and fitted in [21]

the resonances. The quantities to be determined are the parameters $c_{n}, \delta$, and the thresholds $s_{n}$. Following [21], we set $n=1,2, x$ and $s_{1}=\left(m_{\pi}+m_{N}\right)^{2}=1.16 \mathrm{GeV}^{2}$, $s_{2}=2.44 \mathrm{GeV}^{2}$ and $s_{x}=11.7 \mathrm{GeV}^{2}$.

Other parameters of the trajectory obtained in the fit are summarized below: $\alpha(0)=-0.41, \delta=-0.46 \pm 0.07$, $c_{1}=0.51 \pm 0.08, c_{2}=4.0 \pm 0.8$, and $c_{x}=(4.6 \pm 1.7) \cdot 10^{3}$. Taking the central values of these parameters, we obtain the following values for the $\lambda^{\prime} \mathrm{s}: \lambda_{1}=0.846, \lambda_{2}=2.082$, $\lambda_{x}=11.177$.

The fit is fairly good: $\chi^{2} /$ d.o.f $=1.15$, see Figs. 4 and 5 . In the mass range where the parameters of the trajectory were fitted to the data, i.e. $M_{X}^{2} \leq 8 \mathrm{GeV}^{2}$, this is the most realistic proton trajectory we know from the literature. Nevertheless, care should be taken, if it is used outside this range. As long as we are within our applicability range, the sum over resonances in Eq. (13) is restricted to 4 resonances $(n=0,3)$, but, in the imaginary part of the transition amplitude, Eq. (16), we consider the contributions only from three of these resonances, since the imaginary part vanishes for the lowest resonance, i.e. for the proton, $n=0, \operatorname{Im} \alpha=0$, producing an infinitely narrow and high peak.

The elastic contribution $p P \rightarrow p P$ will be discussed in the next section, see also $[27,28]$. However, it can be assumed that, outside the elastic peak, $2 \mathrm{GeV}^{2} \leq M_{X}^{2} \leq$ 


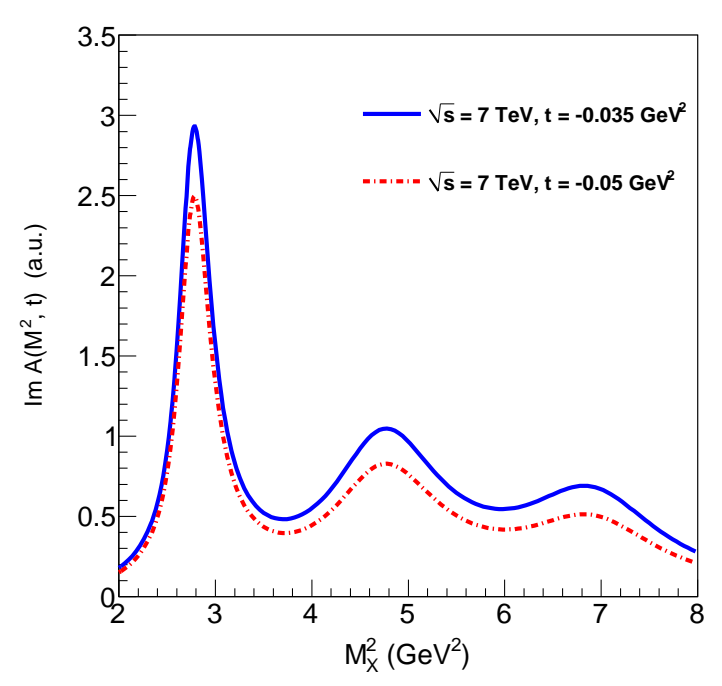

Fig. 6. Imaginary part of the amplitude $A\left(M_{X}^{2}, t\right)$, Eq. (20)

$8 \mathrm{GeV}^{2}$, this distribution can be neglected, because the dominant part come from the nearest resonance.

Thus, we obtain

$\operatorname{Im} A\left(M_{X}^{2}, t\right)=$

$=\sum_{n=1,3} \frac{[f(t)]^{2(n+1)} \operatorname{Im} \alpha\left(M_{X}^{2}\right)}{\left(2 n+0.5-\operatorname{Re} \alpha\left(M_{X}^{2}\right)\right)^{2}+\left(\operatorname{Im} \alpha\left(M_{X}^{2}\right)\right)^{2}}$.

Note that the contribution from each subsequent resonance of the proton trajectory is suppressed by a factor $f(t)^{2}$ as compared with the previous one.

Apart from the well-established proton trajectory, with a sequence of four particles on it, there is a prominent resonance $I=1 / 2, \quad J=1 / 2^{+}$with a mass of 1440 $\mathrm{MeV}$, known as the Roper resonance. It is wide, the width being nearly one quarter of its mass. The Roper resonance may appear on the daughter trajectory of $N^{*}$ treated above, although its status is still disputable (for the more detailed discussion, see [9]).

\section{Results}

Figure 6 shows the behavior of the imaginary part of the transition amplitude, Eq. (20), proportional to the transition form factor as in Fig. 2, or lower vertex in Fig. 1 (right panel). It shows the resonance structure corresponding to the proton trajectory, to be translated into the cross sections via Eq. (7), with the results shown below. One can see that the imaginary part of the transition amplitude decreases with growing $|t|$ due to the dipole form factor $(f(t))^{2(n+1)}$. Furthermore, for each fixed $t$, the relative contribution of higher resonances decreases, because of the suppression factor $f(t)^{2}$ for every subsequent resonance, see Eqs. (13) and (16).

Our final expression for the double differential cross section reads

$$
\begin{aligned}
& \frac{d^{2} \sigma}{d t d M_{X}^{2}}= \\
& =A_{0}\left(\frac{s}{M_{X}^{2}}\right)^{2 \alpha_{P}(t)-2} \frac{x(1-x)^{2}\left[F^{p}(t)\right]^{2}}{\left(M_{x}^{2}-m^{2}\right)\left(1+\frac{4 m^{2} x^{2}}{-t}\right)^{3 / 2}} \times \\
& \times \sum_{n=1,3} \frac{[f(t)]^{2(n+1)} \operatorname{Im} \alpha\left(M_{X}^{2}\right)}{\left(2 n+0.5-\operatorname{Re} \alpha\left(M_{X}^{2}\right)\right)^{2}+\left(\operatorname{Im} \alpha\left(M_{X}^{2}\right)\right)^{2}}
\end{aligned}
$$

Its overall normalization depends on two factors, namely $\beta$, Eq. (7), and $a$, Eq. (13). But, since they do not appear separately, we have combined them in a single factor, $A_{0}=a \beta^{4} / 4 \pi$, to be fitted to the data.

The data on integrated SDD cross sections for various $s$ are available from [1-3] (see also [29]). Unfortunately, the data points are not sufficient to fix this norm unambiguously and to discriminate uniquely the resonance contribution from the background.

To calculate the integrated SDD, we first take into account the contribution from the resonance region. This is done by integrating Eq. (7) with respect to the squared momentum transfer $t$ from $-s$ to 0 and with respect to the missing mass $M_{x}$ over the resonance region $2 \mathrm{GeV}^{2}<M_{x}^{2}<8 \mathrm{GeV}^{2}$, where the contributions from the resonances, Eq. (7) dominate. Thus, we eliminate the contributions from the region of the elastic peak, $M_{X}^{2}<2 \mathrm{GeV}^{2}$, that requires a separate treatment (see [30]) and the high missing mass Regge-behaved region. By duality, to avoid "double counting", the latter should be accounted for automatically, provided the resonance contribution is included properly.

The results for the integrated SDD cross section are shown in Fig. 7. Without any contribution from the background, the fits to the data give $A_{0}=1.09$ with $\chi /$ d.o.f. $=46.4$. A better agreement with the data can be obtained by including a constant background, i.e. by adding a fitting parameter $b$ to the integrated SDD cross section. In this case, the fit gives $\chi /$ d.o.f. $=12.0$, with $A_{0}=0.44$ and $b=3.4 \mathrm{mb}$.

A more advanced model for the background, dual to the Pomeron exchange, was derived in $[14,15]$. Here, the background was parametrized by non-resonance directchannel "pole-like" terms with exotic trajectories dual to 


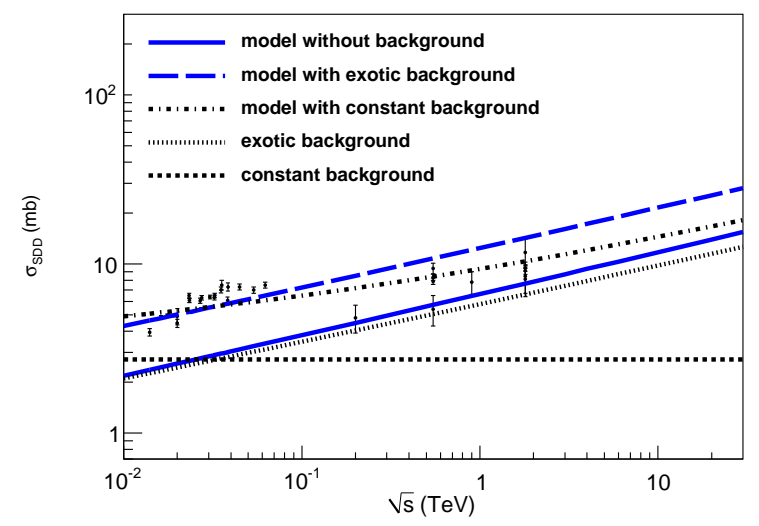

Fig. 7. Predicted integrated SDD cross section as a function of $s$ compared with the experimental data [1-3,32-37]; see also [29]

the Pomeron exchange:

$\left[A\left(s, Q^{2}\right)\right]_{\mathrm{BG}}=G_{E} \frac{f_{E}^{2}\left(Q^{2}\right)}{n_{E}-\alpha_{E}(s)}$,

where the exotic trajectories were chosen in the following form:

$\alpha_{E}(s)=\alpha_{E}(0)+\alpha_{1 E}\left(\sqrt{s_{0}}-\sqrt{s_{0}-s}\right)$.

To prevent any physical resonance, the parameters of the exotic trajectory, Eq. (23), are constrained in such a way that the real part of the trajectory terminates before reaching the first resonance on the physical sheet. An infinite sequence of poles, saturating duality, appears on the non-physical sheet in the amplitude; they do not interfere in the smooth behavior of the background. The exotic form factor is given in the dipole form: $f_{E}^{2}\left(Q^{2}\right)=c_{E}^{2} /\left(c_{E}-Q^{2}\right)^{2}$. The coefficient $G_{E}$ is another normalization parameter. All the exotic parameters, $\alpha_{E}(0), \alpha_{1 E}, c_{E}$, and $G_{E}$, were fitted to the experimental data.

In the same way as in the resonance amplitude, $Q^{2}$ should be replaced by $-t$, and $s$ by $M^{2}$, when applied to SDD calculations. Thus, in such an approach, we obtain our final expression for the double differential cross section:

$$
\begin{aligned}
& {\left[\frac{d^{2} \sigma}{d t d M_{X}^{2}}\right]_{\mathrm{BG}}=} \\
& =A_{0} G_{E}\left(\frac{s}{M_{X}^{2}}\right)^{2 \alpha_{P}(t)-2} \frac{x(1-x)^{2}\left[F^{p}(t)\right]^{2}}{\left(M_{x}^{2}-m^{2}\right)\left(1+\frac{4 m^{2} x^{2}}{-t}\right)^{3 / 2}} \times \\
& \times \frac{\left[f_{E}(t)\right]^{2} \operatorname{Im} \alpha_{E}\left(M_{X}^{2}\right)}{\left(0.5-\operatorname{Re} \alpha_{E}\left(M_{X}^{2}\right)\right)^{2}+\left(\operatorname{Im} \alpha_{E}\left(M_{X}^{2}\right)\right)^{2}} .
\end{aligned}
$$

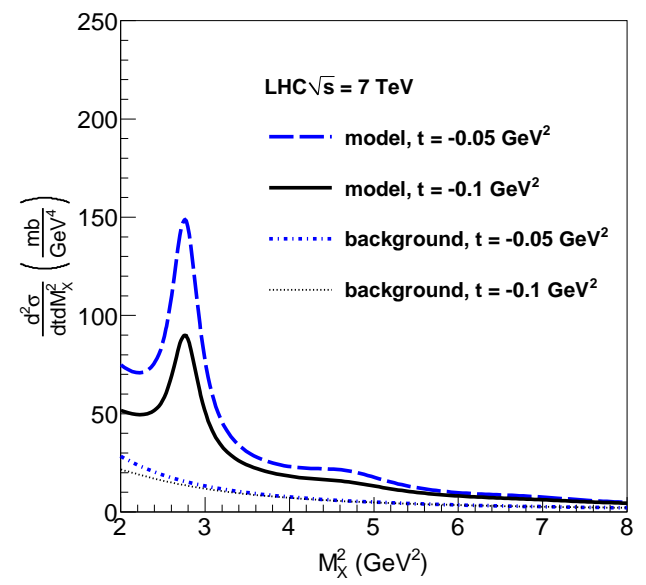

Fig. 8. Double differential cross section of SDD as a function of $M_{X}^{2}$ for several fixed values of $t$ at $\mathrm{LHC} \sqrt{s}=7 \mathrm{TeV}$

To calculate the integrated SDD, we integrate over the squared momentum transfer $t$, and over the missing mass $M_{x}$. However, the BG part should be integrated over the whole $M_{x}^{2}$ physical interval from 0 to $s$.

However, as we can see in Fig. 7, such a background, although physically justified and with extra free parameters, does not improve the fit compared to a simple constant term: the "exotic" background results in $\chi^{2} /$ d.o.f. $=22.3$ for the following values of the fitted parameters: $\alpha_{E}(0)=0.54, \alpha_{1 E}=-0.0031 \mathrm{GeV}^{-1}$, $c_{E}=20.0 \mathrm{GeV}^{2}$ and $G_{E}=65.8$.

Having fixed the parameters of the model and of our "exotic" background, we can now scrutinize the SDD cross section in more details. First, we calculate the double differential cross section, Eq. (7), as a function of the missing mass for several fixed values of the momentum transfer $t$ and two representative LHC energies, 7 and $14 \mathrm{TeV}$. The results of calculations are shown in Figs. 8 and 9.

In Fig. 10, we show the energy dependence of the cross section for $t=-0.05 \mathrm{GeV}^{2}$ and several fixed values $M_{x}$.

Figures 11 and 12 show the differential cross section in $t$ for two representative LHC energies and several fixed values of $M_{X}$. Figure 13 shows the $t$-dependence of the differential cross section integrated over $M_{X}^{2}$. The rise of $\sigma_{\mathrm{SDD}}(s)$ is mainly determined by the supercritical Pomeron intercept $\alpha_{P}(t)$, although it is affected also by the details of the $t-$ and $M_{X}$ dependence.

\section{Conclusions and Perspectives}

Let us briefly summarize the status of the present model and its credibility, including the way its parameters were fixed. As was already mentioned, the normalization con- 


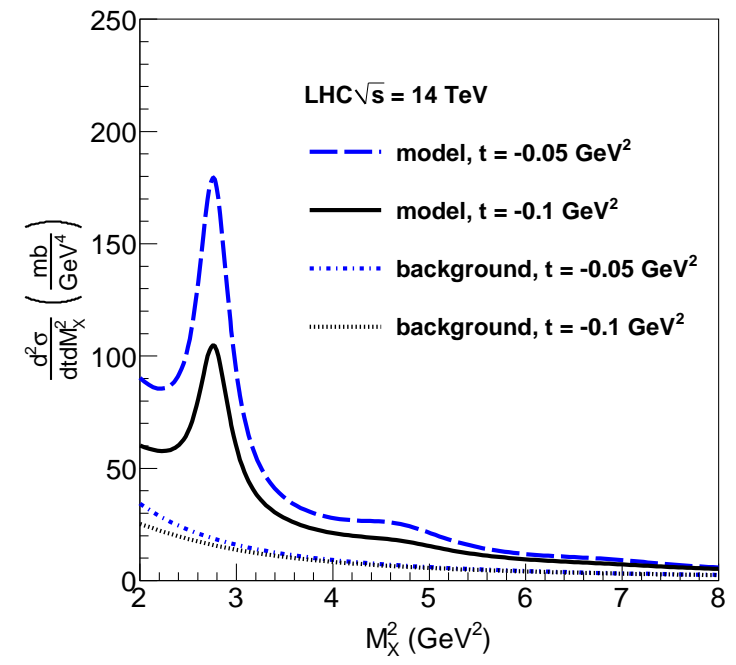

Fig. 9. Double differential cross section of SDD as a function of $M_{X}^{2}$ for several fixed values of $t$ at $\mathrm{LHC} \sqrt{s}=14 \mathrm{TeV}$

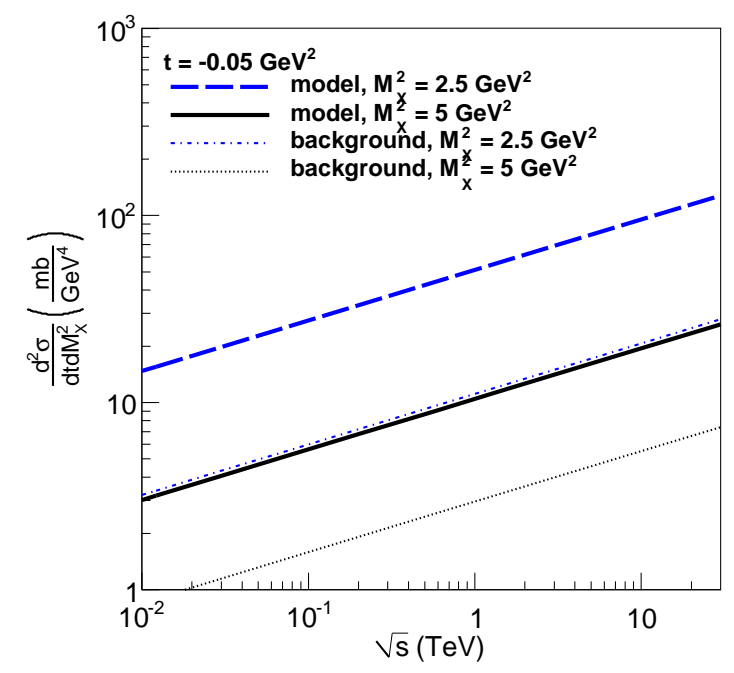

Fig. 10. Double-differential cross section of SDD as a function of $s$ at fixed value of $t$ and $M_{X}^{2}$

stant $\beta$ discussed in Secs. 2 and 3 is absorbed by the overall norm $A_{0}$, together with the parameter $a$; the parameters of the Pomeron trajectory were determined [11] from $p p$ elastic and total cross section data. The form and the values of the parameters of the proton trajectory that plays a crucial role in predicting the $M_{X}$ dependence are fixed by spectroscopic data, see Sec. 5 . Finally, the parameter $a$ is absorbed by the norm $A_{0}$, and it is fixed from the comparison of the calculated SDD cross section with the experimental data, with the following caveat: in the present model applicable at the LHC, only the Pomeron trajectory contributes to the

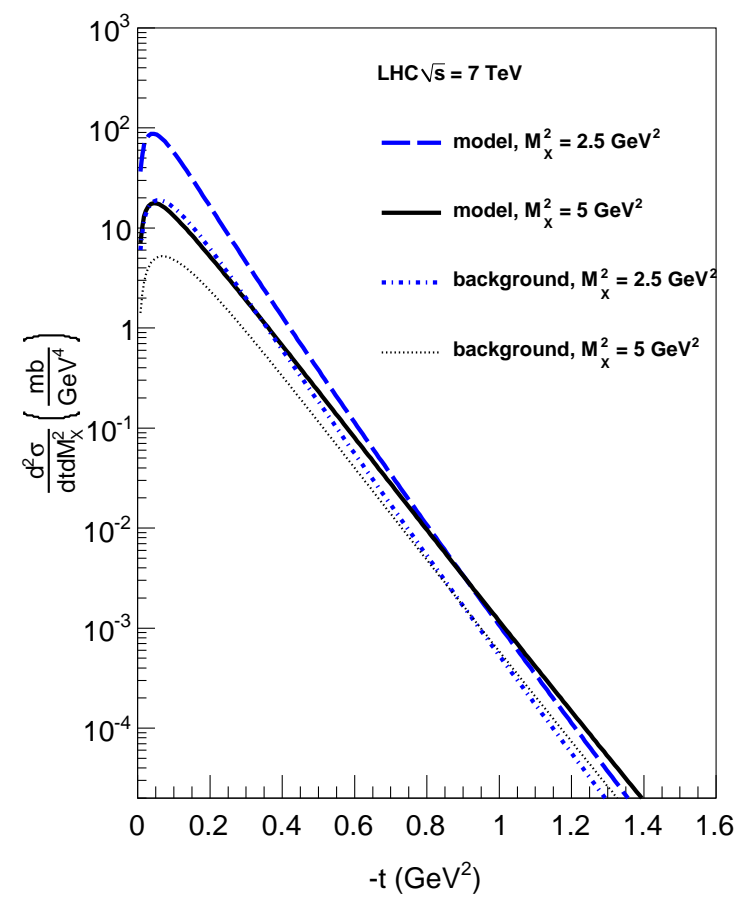

Fig. 11. Double-differential cross section of SDD as a function of $t$ at fixed values of $M_{X}^{2}$ and $\sqrt{s}=7 \mathrm{TeV}$

$t$ channel. At much lower energies (Fermilab, ISR,...), where data are available, secondary non-leading trajectories contribute as well, apart from the Pomeron. They can and should be included in a relevant analysis (fit) of those data.

There is some freedom in the form and weight of the background. Its relative contribution can be normalized to earlier measurements at the ISR or the Fermilab, and anyway its contribution at the LHC is expected to be small, within the experimental error bars. For a better control, we compare our predictions with the experimental data [1-3] and theoretical estimates [10, 16, 29]. In any case, it follows from our model and the fits to the data that the background is fairly large (about 25\% at the LHC), and it interferes constructively at low energies and destructively at high energies. A study of various options for the background in SDD can be found in [30].

The elastic contribution $p p \rightarrow p p$ is usually calculated and measured separately. There is no consistent theoretical prescription of any smooth transition from inelastic to elastic scattering, corresponding to the $x \rightarrow 1$ limit for the structure functions (see [30]).

There is an important point omitted in this short paper, namely unitarity. As is well known (see, e.g., [24]) any simple Regge pole model violates unitarity in the sense that the DD cross section asymptotically grows 


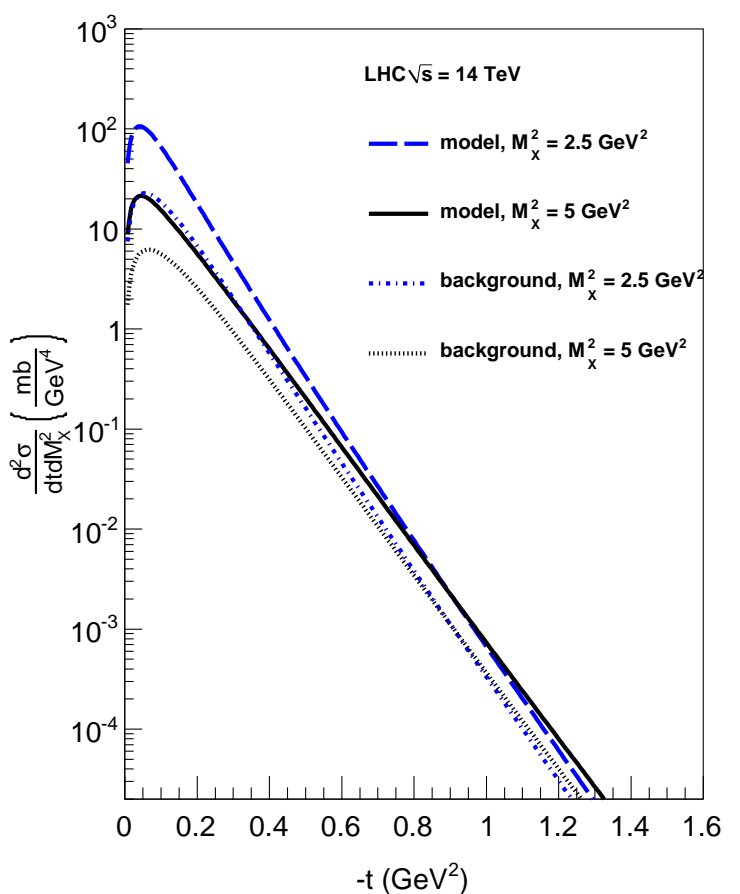

Fig. 12. Double-differential cross section of SDD as a function of $t$ at fixed values of $M_{X}^{2}$ and $\sqrt{s}=14 \mathrm{TeV}$

faster that the total cross section (it is obvious that no partial cross section can overshoot the total cross section). This long-standing problem was cured in various ways, the final answer being still open. In [5,10,16], unitarity is restored by a renormalization procedure. Without entering into details, we only mention that a possible solution of this problem can be found by using a more realistic (and complicated) Pomeron singularity, for example, in the form of a double pole [31].

The model presented in this paper and the calculated cross sections corrected for the efficiencies of relevant detectors will be used [26] in future measurements at the LHC.

\subsection{Perspective of measuring SDD at $\mathrm{LHC}$}

As was already mentioned in Introduction, the prospects of measuring SDD at the LHC are promising, although some details still remain to be settled. For the CMS Collaboration, the SDD mass coverage is presently limited to some $10 \mathrm{GeV}$. Together with the T2 detectors of TOTEM, SDD masses down to $4 \mathrm{GeV}$ could be covered, provided the TOTEM trigger (data acquisition) system will be combined with the CMS ones. ALICE and LHCb have different beam arrangements, but their acceptances for central diffraction (double pomeron exchange) was

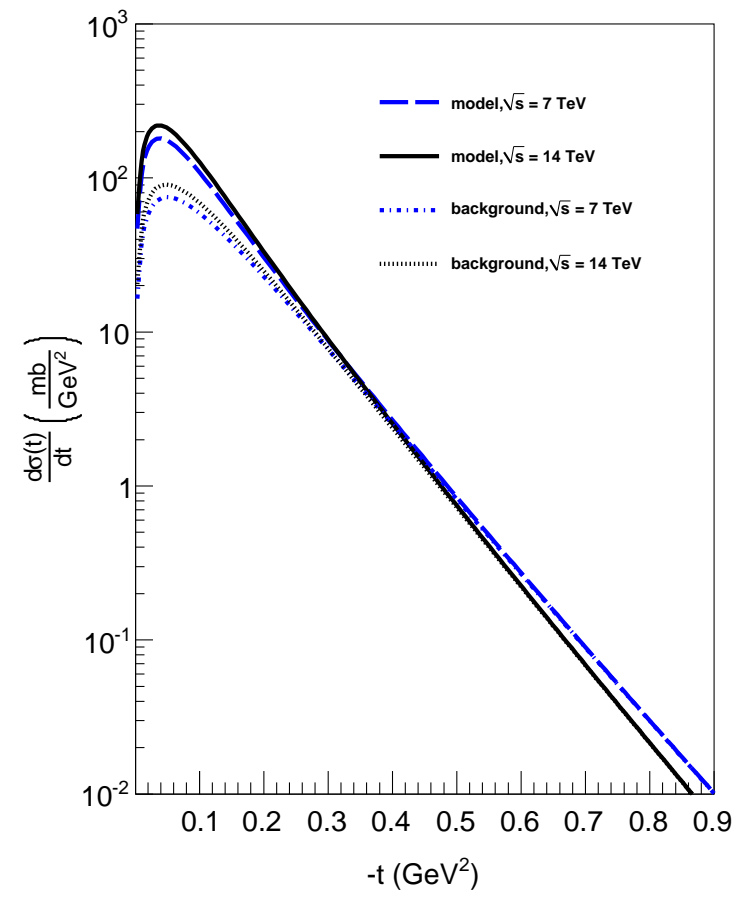

Fig. 13. Differential cross section $d \sigma / d t$ as a function of $t$

also investigated (see, e.g., [4]). Measurements of the SDD events at the LHC are based on: (1) identifying a gap in forward rapidities in conjunction with a veto for any activity on the opposite side of the interaction point or (2) detecting a diffractively scattered proton in a leading proton detector, such as the Roman Pots, and a coincident diffractively excited bunch of particles on the opposite side. The problem with both measurement strategies stems from the incomplete rapidity coverage of the base line detector systems at the LHC: the low-mass $(M<4 \mathrm{GeV})$ diffractively excited states are not seen. Without extra rapidity coverage below $M=4 \mathrm{GeV}$, both approaches to the SDD identification fail. In case of the purely rapidity gap based method, the recorded cross section misses the SDD events with diffractive masses below $4 \mathrm{GeV}$. In case a leading proton is detected on one side of the Intersection Point (IP), it could, in principle, be sensitive to diffractive masses that correspond to the uncertainty in the LHC beam energy. In practice, it is impossible to trigger for these events, and the low-mass SDD events will be missed by this method as well. Detecting the SDD events with high acceptance is essential for determining the total $p p$ cross section in the so-called luminosity independent method based on using the optical theorem. The method is based on measuring the slope of the elastic cross section, extrapolating the slope to the optical point. Together with the over-all inelastic 
rate (plus the ratio between the inelastic and elastic forward scattering amplitudes), the total $p p$ cross section is obtained. The main uncertainty in this evaluation is due to the error in estimating the inelastic $p p$ event rate. As shown in [4], the acceptance of basically all the LHC experiments can be substantially improved by adding forward detector systems (Forward Shower Counters, FSCs) that register secondary interactions within the beam pipe due to the particles - both electrically neutral and charged - emitted at very small scattering angles with respect to the beam direction. With the addition of FSCs, the rapidity coverage of an LHC experiment can be extended down to SDD masses of the order of $1.2 \mathrm{GeV}$, i.e. down to the dominant $N^{*}$ states. FSCs are being currently installed in ALICE and CMS detectors, and they will provide the necessary added coverage of small-mass forward systems at the LHC.

We thank Dino Goulianos, Risto Orava and Francesco Paccanoni for useful discussions. O.K. is grateful to Rainer Schicker and the staff of the Bogolyubov ITP, in particular to A.G. Zagorodny, for their support. The work of V.K.M. is partly supported by the contracts FIS2008-01661 from MICINN (Spain), by the Generalitat de Catalunya contract 2009SGR-1289, and by the European Community-Research Infrastructure Integrating Activity "Study of Strongly Interacting Matter" (HadronPhysics2, Grant Agreement n. 227431) under the Seventh Framework Programme of EU. L.J. was supported by the National Ac. Sc. of Ukraine, Division of Astronomy and Physics, under the Grant "Matter under Extreme Condition". The work of L. Jenkovszky was supported also by the Hungarian Science Abroad Program.

1. R.D. Schamberger et al., Phys. Rev. Letters, 34 (1975) 1121 .

2. M. Albrow et al., Nucl. Phys. B 1081 (1976); J.C.M. Armitage et al., Nucl. Phys. 194365 (1982).

3. R.D. Schamberger et al. Phys. Rev. 171268 (1978).

4. M. Albrow, A. De Roeck, V. Khoze, J. Lamsa, E. Norbeck, Y. Onel, Risto Orava, and M.G. Ryskin, arXiv:0811.0120 [hep-ph].

5. K. Goulianos, Phys. Rep. 101169 (1983).

6. D.P. Roy and R.G. Roberts, Nucl. Phys. B 77240 (1974).

7. G.A. Jaroszkiewicz and P.V. Landshoff, Phys. Rev. D 10 170 (1974).

8. A. Donnachie and P.V. Landshoff, Nucl. Phys. B 244322 (1984).
9. L.L. Jenkovszky, O.E. Kuprash, J.W. Lamsa, V.K. Magas, and R. Orava, arXiv:1011.0664 [hep-ph], to appear in PRD.

10. K. Goulianos and J. Montanha, Phys. Rev. D 59114017 (1999).

11. S. Donnachie, G. Dosch, P. Landshoff, and O. Nachtmann, Pomeron physics and $Q C D$ (Cambridge Univ. Press, Cambridge, 2002); P.V. Landshoff, The total cross section at the $\mathrm{LHC}$, Lectures at the Copanello Summer School, Calabria, 2007, Acta Phys. Pol. B 392063 (2008).

12. R. Fiore, L. Jenkovszky, R. Orava, E. Predazzi, A. Prokudin, and O. Selyugin, Int. J. Mod. Phys. A 242551 (2009); arXiv:0812.0539 [hep-ph].

13. L.L. Jenkovszky, O.E. Kuprash, J.W. Lämsä, V.K. Magas, and R. Orava, Dual-Regge Approach to High-Energy, Low-Mass Diffraction Dissociation, hep-ph/1011.0664.

14. R. Fiore et al., EPJ A 15 (2002) 505, hep-ph/0206027.

15. R. Fiore et al., Phys. Rev. D 69014004 (2004), hep$\mathrm{ph} / 0308178$.

16. K. Goulianos, Phys. Letters B 358379 (1995).

17. L. Jenkovszky, V.K. Magas, and E. Predazzi, EPJ A 12 361 (2001), hep-ph/0110374.

18. N.A. Amos et al., Phys. Lett. B 247127 (1990).

19. A.I. Bugrij et al., Fortschr. Phys. 21, 427 (1973).

20. V. K. Magas, Phys. Atom. Nucl. 68, 104 (2005); [arXiv:hep-ph/0404255]; arXiv:hep-ph/0411335; PoS D IFF2006, 051 (2006) [arXiv:hep-ph/0611119].

21. R. Fiore, L.L. Jenkovszky, F. Paccanoni, and A. Prokudin, Phys. Rev. D 70054003 (2004); hep-ph/0404021.

22. Data Particle Group Collaboration, C. Amsler et al., Phys. Lett. 6671 (2008).

23. F. Ravndal, Phys. Lett. 37B, 300 (1971).

24. P.D.G. Collins, An Introduction to Regge Theory and High Energy Physics (Cambridge Univ. Press, Cambridge, 1977).

25. C. Carlson and N.C. Mukhopadhyay, hep-ph/9801205.

26. L. Jenkovszky, O. Kuprash, J.W. Lamsa, and Risto Orava, in preparation.

27. A. Bodek et al., Phys. Rev. D 201471 (1979).

28. I. Niculescu, Ph.D. Thesis (Hampton University, May 1999).

29. A.A. Arkhipov, hep-ph/0012349 v1.

30. L.L. Jenkovszky, V.K. Magas, O.E. Kuprash, Proceedings of the International Workshop on Diffraction in HighEnergy Physics (Diffraction 2010), Otranto, Lecce, Italy, September 10-15, 2010, to be published by the American Institute in Physics (AIP). 
31. L.L. Jenkovszky, E.S. Martynov, and F. Paccanoni, Proceedings of the "HADRONS-96" Conference (Novy Svet, Crimea, 1996).

32. B.E. Ansorge et al., Z. Phys. C 33, 175 (1986).

33. G.J. Alner et al., (UA5) Phys. Rep. 154, 247 (1987).

34. F. Abe et al., CDF Collaboration, Phys. Rev. D 505535 (1994).

35. R. L. Cool, K. Goulianos, S.L. Segler, H. Sticker and S.N. White, Fermilab E396, Phys. Rev. Lett. 47701 (1981).

36. D. Bernard et al., Phys. Lett. B 186227 (1987).

37. N. A. Amos el al., (E710 Collaboration), Phys. Lett. B 301313 (1993).

Received 18.02.11
ДИФРАКЦІЙНА ДИСОЦІАЦІЯ НА МАЛІ МАСИ НА LHC. РОЛЬ ФОНУ

Л.Л. Єнковсъкий, О.Є. Купраш, В.К. Магас

P е $з$ ю м е

В роботі представлено дуальну модель із нелінійною траєкторією Редже у масовому каналі. Використано фон, заснований на екзотичній траєкторії у прямому каналі, який було розроблено та застосовано раніше для опису інклюзивного перерізу електрон-протонного розсіяння в області нуклонних резонансів. Параметри моделі визначено з апроксимації результатів раніших експериментів. Зроблено передбачення перерізів дифракційної дисоціації на малі маси $\left(2<M_{X}^{2}<8 \Gamma^{2}\right)$ для енергій LHC. 